\title{
Entre la ruta y la empresa: estrategias de profesionalización de la actividad agropecuaria durante del agronegocio (Córdoba, fines del siglo XX)
}

\author{
Between the route and the company: strategies of professionalization of the agricultural activity during the \\ agribusiness (end of the 20th century) \\ Gabriel Fernando Carini \\ Centro de Investigaciones Históricas de la Universidad Nacional de Rio Cuarto [CIH-UNRC] Centro \\ de Investigaciones de la Facultad de Filosofía y Humanidades de la Universidad Nacional de Córdoba \\ Becario del CONICET, Argentina \\ gcarini@hum.unrc.edu.ar
}

\section{Resumen:}

El objetivo del presente artículo es analizar las transformaciones en las dinámicas institucionales de las asociaciones rurales durante la vigencia del agronegocio. Sostenemos que los imperativos del nuevo modelo productivo tensionaron los perfiles institucionales que históricamente caracterizaron a las asociaciones rurales. Así, la dirigencia rural emprendió una serie de innovaciones en su estructura organizacional, que implicaron una nueva concepción de la labor gremial, favoreciendo aspectos ligados a una racionalidad empresaria. Exploraremos estas cuestiones a partir del análisis de una entidad de primer grado de la pampa cordobesa, lo que nos permitirá una mirada más exhaustiva de los procesos estudiados.

PALABRAS ClaVE: Asociaciones rurales, Racionalidad empresaria, Agronegocio, Dinámicas institucionales.

\section{Abstract:}

The objective of this article is to comment on the transformations in the institutional dynamics of rural associations during the lifetime of agribusiness. We maintain that the imperatives of the new productive model stressed the institutional profiles that historically characterized rural associations. Thus, the rural leadership undertook a series of innovations in its organizational structure that implied a new conception of union work, favoring aspects linked to an entrepreneurial rationality. We will explore these questions based on the analysis of a first degree entity from the pampas of Cordoba, which will allow us to take a more exhaustive look at the processes studied.

KEYWORDS: Rural associations, Business rationality, Agribusiness, Institutional dynamics.

\section{INTRODUCCIÓN}

A partir de la década de 1990 comenzaba a percibirse como central la incorporación de diversos saberes para optimizar los resultados económicos de las empresas agropecuarias. De esta forma, se allanó el camino hacia el predominio de una lógica empresarial que supuso nuevas modalidades de integración de la producción agropecuaria (que trasvasaron lo sectorial) y la necesidad de otorgar flexibilidad tanto a la planificación y ejecución de las tareas agrícolas como a las de gestión de las explotaciones agropecuarias. ${ }^{1}$ Estas mutaciones implicaron la transformación de la actividad agropecuaria y la hicieron cada vez más dependiente de los conocimientos científicos y de los insumos, pero también del management y las estrategias de producción, indispensables para sortear las difíciles y ambivalentes condiciones por las que atravesaba el sector. Estos lineamientos cambiaron el manejo económico de las empresas agropecuarias: no solo produjeron un incremento en las escalas necesarias para mantenerse dentro del proceso productivo, sino que resignificaron las relaciones entre los actores a lo largo del mismo (C. entre otros: Murmis, 1998; 
Teubal et al., 2005; Alapín, 2008; Gallacher, 2010; Gras y Hernández, 2009; 2013 y 2016; Hernández, 2009).

Lo que nos interesa destacar es que este conjunto de rupturas tuvo profundas incidencias sobre las dinámicas internas y externas de las asociaciones de productores. En este sentido, diversas investigaciones han enfatizado en la vigencia de una nueva institucionalidad en el agro argentino, que estuvo encarnada en las asociaciones de carácter técnico, es decir, aquellas cuyo principal objetivo era promover determinadas formas organizativas, cultivos o técnicas específicas. Estas entidades -algunas de las cuales surgieron durante el período considerado- fueron, en la perspectiva de los investigadores, las principales difusoras (y legitimadoras) del nuevo paradigma productivo. ${ }^{2}$ Sobre la base de esa caracterización se construyeron visiones dicotómicas referidas a la función desempeñada por las asociaciones de productores. Se reservó, así, la función exclusivamente gremial para aquellas que habían surgido durante períodos anteriores y que respondían a las necesidades coyunturales de otros modelos productivos. Incluso se propuso una suerte de división de tareas que no implicó la competencia por las bases sociales, sino una suerte de complementación (Gras, 2009). Es decir, las "nuevas" entidades condenaron a una situación de "marginalidad" y/o "obsolescencia" a las asociaciones gremiales, devenidas en tradicionales (Lattuada, 2006).

Más allá de estas discusiones, consideramos necesario volver nuestra mirada sobre las dinámicas institucionales de las asociaciones gremiales de productores a los fines de revisar qué respuestas estructuraron frente al contexto productivo. En este sentido, sostenemos que, lejos de asumir una actitud pasiva, la dirigencia gremial de las asociaciones rurales internalizó los imperativos del agronegocio e imprimió una serie de mutaciones en los perfiles institucionales de las entidades reivindicativas, lo que -sin desplazar su función de defensa de los intereses de sus bases sociales- promovió el fortalecimiento de los rasgos empresarios de sus bases sociales, tanto como estrategia frente a la competitividad impuesta por el agronegocio como frente a la presión ejercida por la emergencia de nuevos referentes en el entramado asociativo del agro argentino.

El presente artículo se encuentra organizado en tres apartados. En el primero ofrecemos algunas precisiones sobre el referente empírico del objeto de estudio. En este punto, nos interesa explicitar algunas elecciones de orden metodológico que justifican la elección del mismo. En el segundo historizamos el proceso por medio del cual la dirigencia rural de la entidad analizada generó una redefinición en su perfil institucional, a los fines de adecuarlo a los imperativos del agronegocio. Nos concentraremos en dos cuestiones. Por un lado, comentaremos los cambios introducidos en el modelo organizativo de la entidad, refiriéndonos exclusivamente a la figura de las comisiones asesoras. Por el otro lado, avanzaremos en la descripción de las actividades tendientes a mejorar la performance económica de sus bases sociales, generadas a partir de esos espacios institucionales.

\section{Algunas precisiones metodológicas SOBRe UN ABORDAJE POSIBLE DE LAS ASOCIACIONES RURALES}

El estudio de las asociaciones de productores constituye una temática de largo aliento en el campo de las ciencias sociales. En ese conjunto de producciones se puede observar el predominio de al menos dos tipos de abordajes. Por un lado, encontramos aquellos que podríamos denominar como institucionalizantes, generalmente elaborados por sus asociados, que propusieron miradas apologéticas, con el objetivo de fijar la memoria de las entidades y/o exaltar la figura de determinados líderes. También dentro de este grupo, pero en un polo diametralmente opuesto, desde la segunda mitad del siglo XX proliferaron trabajos que -consustanciados con la programática de ciertos partidos políticos o doctrinas ideológicas- construyeron una imagen de los terratenientes pampeanos (especialmente de los grandes ganaderos) como una clase que afincaba su riqueza en el control de grandes extensiones de tierra y en prácticas económicas especulativas que permitían la reproducción de su capital sin asumir riesgo empresario. Por otro lado, desde finales del siglo XX, 
inscritos explícita o implícitamente en los postulados del neocorporativismo se propusieron abordajes más integrales -apegados a los cánones metodológicos de la labor de la investigación-que ofrecieron explicaciones sobre las dinámicas internas de las entidades, las formas de resolución de los conflictos, la composición socioeconómica de sus bases sociales, así como las dinámicas externas referidas no solo a las modalidades de mediación política sino también a la posibilidad de acciones concertadas entre el arco del empresariado en general y de las asociaciones agrarias en particular (Carini, 2018).

Estas investigaciones centraron su mirada en entidades que asumían una representación "nacional", como la Sociedad Rural Argentina (SRA), la Federación Agraria Argentina (FAA) y, en menor medida, de la Confederación de Asociaciones Rurales de Buenos Aires y La Pampa (CARBAP), y de las Confederaciones Rurales Argentinas (CRA). En este sentido, consideramos que es necesario realizar estudios que apelen a la reducción de la escala para permitir un abordaje más exhaustivo de las transformaciones que supuso la vigencia del agronegocio en las lógicas institucionales de las entidades gremiales. Cabe advertir que este trabajo no pretende ser representativo o constituirse en una muestra de casos más generales, sino que intenta abordar singularidades para demostrar cómo esas especificidades pueden, en todo caso, contradecir y deconstruir los casos globales, más que representarlos fielmente a la manera positivista clásica. No obstante, esto no implica abandonar la pretensión de generalidad a partir de los estudios micro, puesto que los mismos pueden contribuir a revelar aspectos fundamentales sobre los fenómenos generales. A los fines de indagar estas cuestiones, tomaremos como objeto de análisis la Sociedad Rural de Río Cuarto. En la elección de este referente empírico se contemplaron diferentes cuestiones de orden teórico y metodológico. En primer lugar, se contempló el perfil institucional de la SRRC - es decir, todos aquellos aspectos ligados a su estructura interna y externa-, que constituye un tipo ideal de entidad gremial. Desde su creación a principios del siglo $\mathrm{XX}$, y con mayor vigor desde su consolidación a mediados de la década de 1930, se encontraba abocada estrictamente a la defensa de los intereses de los productores ganaderos asociados a ella, y poseía una frágil estructura de servicios que se restringía a la provisión de guías ganaderas. Además, se encontraba (y encuentra) adherida a la Confederación de Asociaciones Rurales de la Tercera Zona (CARTEZ) y, por su intermedio, a las Confederaciones Rurales Argentinas (CRA). Todas instancias supralocales de articulación de intereses agrarios (Ver: Carini, 2015, 2017). ${ }^{4}$

En segundo lugar, nos interesa enfocarnos en las capas de productores previamente "incluidas", es decir, los que ya habían entrado en el proceso de capitalización y tecnificación (Murmis, 1998, p. 234). Estos sujetos sociales son los que conforman la base social de la SRRC (Carini, 2014). Este sector se compone de un conjunto de sujetos sociales que constituyen un factor decisivo en el mantenimiento de formas de vida rural modernizada, pero con presencia local. Estos sujetos han recibido diferentes denominaciones, que enfatizan en la conjunción entre diversos aspectos, en especial en lo relativo a la incorporación de tecnologías, a las dimensiones y modalidad de gestión de sus explotaciones, y a los vínculos de sus explotaciones con diferentes territorios: productores tradicionales capitalizados (Barsky y Gelman, 2009, p. 494), productores tradicionales reconvertidos (Flood, 2005, p. 146), o productores territorializados (Gras y Hernández, 2013, p. 53). Estos productores conservan bajo su propiedad los espacios productivos y mantienen el control sobre el proceso productivo, lo que no excluye la contratación de maquinarias ni la ampliación de superficies productivas mediante el arriendo. Puede tratarse de empresas de mediana o gran escala con cierto grado de diversificación territorial, por lo que poseen vínculos con distintos territorios, es decir, que dependen de diversas dinámicas locales. Fue, precisamente, en las franjas medias y superiores de la estructura agraria que algunos investigadores han observado un corrimiento hacia formas más empresariales en los perfiles socioeconómicos que históricamente caracterizaron a los productores comprendidos en ese sector (Gras, 2009; Gras, 2010; Hernández, 2009; Gras y Hernández, 2009; Gras y Hernández, 2013). 


\section{UN NUEVO HORIZONTE ASOCIATIVO: LA CONCERTACIÓN DE NEGOCIOS Y LA PROMOCIÓN DE NUEVAS FORMAS ASOCIATIVAS}

El presente apartado tiene como objetivo dar cuenta del proceso de profesionalización de los productores de la pampa cordobesa impulsado por la dirigencia de la SRRC. Las diferentes acciones generadas con la finalidad tanto de mejorar las prácticas agronómicas y empresariales como de acercar a sus asociados novedosas pautas, instrumentos y habilidades para operar dentro y fuera del proceso productivo comprendieron la estructuración de diversas instancias institucionales. Un primer ámbito privilegiado fueron las ferias anuales de invierno. El certamen ganadero que constituía la marca identitaria de la SRRC ---que al mismo tiempo era su principal fuente de ingreso- registró un viraje que, sin modificar su función esencial, indicó un intento de adecuación al nuevo contexto económico y productivo trazado por el emergente agronegocio. Se propició la actualización tecnológica y productiva por medio de conferencias y cursos a cargo de diferentes especialistas. Una segunda instancia significativa para la transformación de los perfiles socioeconómicos de las bases sociales de la entidad fue el Ateneo Juvenil. La participación de las juventudes no constituía una novedad en la institución y se remontaba a la década de 1960, pero fue en el tránsito a la década de 1990 que adquirió una nueva dimensión. Se asistió a un reforzamiento del compromiso de los jóvenes con la institución, que se tradujo en la organización de eventos que no solo garantizaron la reproducción ideológica de los ideales de la SRRC y la formación de los futuros cuadros dirigenciales, sino que también favorecieron la recepción de los saberes que la configuración del nuevo modelo productivo consideraba como social y productivamente valiosos (Ver: Carini, 2018).

También existieron otras dos instancias desde las que se procuró -de forma más concreta- ofrecer a los asociados de la entidad recursos para fortalecer su desempeño económico. Hubo un sostenido esfuerzo institucional, desarrollado principalmente a partir de las cámaras asesoras de la entidad, por adicionar a las funciones básicas de defensa gremial la oferta de servicios. En ese marco, la dirigencia desarrolló un conjunto de actividades que profundizaron los lineamientos anteriormente señalados. Nuevamente, los saberes asociados a técnicas, recursos y capacidades centrales en la nueva concepción de lo agropecuario constituyeron el eje del accionar institucional. Este accionar privilegió lo atinente a la asesoría legal y contables, a la adopción de la siembra directa y a los recaudos de sanidad animal para asegurar la comercialización de los productos pecuarios. Por último, esas acciones también se evidenciaron, por una parte, en la difusión y capacitación sobre el uso de instrumentos financieros que facilitaban las transacciones comerciales en los mercados internacionales, y, por otra parte, se realizaron acciones que propiciaron la realización de negocios, poniendo en contacto empresas y empresarios nacionales e internacionales con los productores locales. En todas esas propuestas tuvieron una intervención privilegiada actores individuales y colectivos asociados a la nueva institucionalidad agraria nacida al calor del agronegocio, comprometidos tanto con la divulgación de sus implicancias como con la legitimación de sus pilares. Sobre estas acciones nos detendremos en los apartados siguientes.

\subsection{La transformación del modelo organizativo como vía para la reconversión}

La SRRC, como otras asociaciones de productores, poseía un modelo organizativo de escasa complejidad. Su diagrama institucional preveía una comisión directiva que fuera el órgano de conducción de la entidad. Estaba integrada por el presidente, un vicepresidente $1^{\circ}$ y un vicepresidente $2^{\circ}$, un secretario y un prosecretario, y un tesorero y un protesorero, que duraban dos años en su mandato y eran reelegibles. Se completaba con siete vocales titulares y cuatro vocales suplentes. La mitad de los vocales se renovaba anualmente en asamblea general ordinaria. Además, había un tribunal revisor de cuentas, que estaba integrado por tres asociados. A esos espacios se les sumaba el funcionamiento de la Asamblea General, que era la principal autoridad decisoria y fiscalizadora de la SRRC. En ella participaban todos los asociados, sin 
discriminar por antigüedad o por otros motivos, como, por ejemplo, el cumplimiento de la cuota societaria. La Asamblea General podía revestir carácter ordinario o extraordinario. La primera era convocada al cierre del balance anual, en el mes de noviembre, y tenía lugar en el mes de diciembre. Entre sus potestades se encontraba aprobar el balance y la memoria anual y la renovación total o parcial de la Comisión Directiva.

Esta estructura comenzó a complejizarse a mediados de la década de 1990. Desde su refundación en 1935, el recambio de autoridades se había realizado de forma consensuada sin que se presentasen listas alternativas, cuestión que no resulta novedosa, dada la homogeneidad interna (económica, social y, en muchas ocasiones, ideológica) que presentan estas asociaciones y que garantiza no solo la estabilidad en los cuadros dirigenciales, sino también la escasa o nula disidencia (Palomino, 1988, p. 65). La elección de 1994 rompió con la tradición de listas de unidad debido a que no se logró llegar a un consenso sobre la distribución de los primeros nueve cargos (Archivo Histórico Municipal de Río Cuarto -AHMRC-, Hemeroteca -H-, Puntal -P-, lunes 31/10/1994, p. 24). En esa oportunidad, las discrepancias giraron en torno a las formas de concebir la actividad gremial. La lista oficialista Voz Rural cuestionaba el carácter cerrado, "elitista”, que se le había dado a la entidad en las gestiones anteriores y que se había tratado de revertir a partir de la incorporación de "auténticos productores". Esto hacía referencia al esfuerzo que esa línea de gestión había puesto por revitalizar el perfil ganadero de la institución, tratando de asociar a la mayor cantidad de productores pecuarios posibles sin distinción de sus escalas productivas o sus perfiles de corte empresarial. Asimismo, se criticaba la filiación política partidaria que muchos integrantes de la lista opositora poseían, ya que se presentaban como un grupo "apolítico" que "representa[ba]n al partido del campo" (AHMRC, H, Suplemento Agropecuario -SA-, viernes 18/11/1994, p. 3). El proyecto de Voz Rural, encabezado por Raúl Manelli y que postulaba a Néstor Geymonat para el cargo de presidente, acentuaba además el tradicional perfil gremial de la entidad y proponía como política institucional profundizar tanto la canalización de los conflictos (y resolución de los problemas de las bases sociales) a través de CARTEZ y CRA, como la participación de la SRRC en esas instancias asociativas, ya que en ese momento contaba con la presidencia y tesorería de CARTEZ (AHMRC, H, SA, viernes 02/12/1994, pp. 2-3). Por su parte, Alternativa Ruralista, lista liderada por Víctor Tonello, que resultó victoriosa, presentaba un proyecto con un componente fuertemente vinculado a la profesionalización del trabajo agropecuario. Se proponía un "nuevo estilo de acción gremial" que consistía en mejorar la situación socioeconómica de los asociados por medio de una constante actualización y capacitación. En esa propuesta adquirió centralidad la estructuración de servicios para el asociado, que se canalizaría a través de un trabajo coordinado entre la Comisión Directiva, el Ateneo Juvenil y las cámaras asesoras. Estas últimas contaban con el apoyo de profesionales ligados a la Universidad Nacional de Río Cuarto, los grupos CREA, el SELSA y el INTA, entre otros organismos especializados (AHMRC, H, P, domingo 04/12/1994, pp. 4-5).

En ese marco, los dirigentes rurales electos emprendieron una reforma estatutaria que creó nuevos espacios institucionales tendientes a renovar la acción gremial de la entidad. Entre estas innovaciones se incorporó a la Comisión Directiva a los representantes de las filiales y al presidente del Ateneo, quienes a partir de ese momento contaron con la posibilidad de una participación más activa en sus deliberaciones (Cfr.: SRRC, Estatutos, año 1988; AHMRC, H, P, miércoles 09/11/1988, p. 22; SRRC, Estatutos, año 1995). Si bien estos espacios no constituían una novedad -dado que en 1988 una reforma previa les otorgó funcionamiento formal-, constituyeron una instancia pensada para fortalecer la presencia gremial de la entidad. Una mención especial merece la participación de los jóvenes en la entidad puesto que asumieron un rol significativo en la promoción de actividades asociadas al nuevo paradigma productivo, en particular, al de la siembra directa (ver: Carini, 2017 y 2018a). Fue también a partir de esta conducción que adquirió un renovado interés no solo la necesidad de comunicar el quehacer institucional y las problemáticas del sector, sino también la necesidad de brindar herramientas para renovar las modalidades de gestión de las unidades productivas. Esa tarea la asumió el jefe de prensa de la SRRC por medio de la edición de una columna institucional, denominada "Información Ruralista”, en el suplemento agropecuario “Tranquera Abierta” del diario Puntal 5 . 
A estas acciones se le sumó la creación de las cámaras asesoras, que reunían a los productores asociados a la entidad en grupos más reducidos -alrededor de diez miembros- vinculados a temáticas o intereses específicos. Funcionaban independientemente de la comisión directiva, tenían su propio día de reunión y elegían un representante en calidad de coordinador y "vocero". Su rol principal era el de asesorar a la comisión directiva y constituirse en el nexo con técnicos y profesionales del sector. Asimismo, en ocasiones eran las encargadas de estructurar actividades concretas dentro de las dos ferias anuales de la entidad, por ejemplo, la cámara de lechería organizaba la Jornada Nacional de Lechería de la feria de otoño; la de granja y producciones alternativas, las Jornadas de Apicultura. A lo largo del período considerado aparecen menciones a las siguientes cámaras: de ganadería, de agricultura, de granja y producciones alternativas, de lechería, de capacitación o actualización.

Paralelamente, la constitución de las cámaras asesoras buscaba el acercamiento a actores que se consideraban estratégicos para la mejora de "varios aspectos sociales que afecta[ba]n a los hombres de campo", como la Universidad Nacional de Río Cuarto, los grupos CREA, el INTA y la Secretaria de Agricultura, entre otros (AHMRC, H, P, sábado 05/11/1994, p. 20). Integradas en mayoría por asociados de la entidad, debían procurar incorporar a diferentes profesionales y técnicos referentes del quehacer rural dispuestos a colaborar con "acción, ideas y sugerencias" en el accionar de la entidad y "con ello ofrecer nuevos y mejores servicios de gestión y asesoramiento legal, contable y de producción, para los productores" (AHMRC, H, $P$, sábado 05/11/1994, p. 20; AHMRC, H, $S A$, viernes 02/06/1995, pp. 4-5).

Desde esta nueva ingeniería institucional se propició una batería de eventos, capacitaciones y circulación de información asociados a los saberes y prácticas del agronegocio, que introdujeron nuevos elementos a los perfiles institucionales de la entidad. Uno de los puntales de ese accionar estuvo a cargo de la comisión asesora de ganadería. Los objetivos de la misma combinaban los principios más tradicionales de la acción gremial (la defensa de los intereses ganaderos) con nuevas coordenadas más vinculadas a la coyuntura productiva y financiera característica del período que propiciaban la vinculación transectorial y la búsqueda de nuevos canales de comercialización tanto nacionales como internacionales. Así, entre los principios que direccionaron las estrategias de la cámara asesora de ganadería, se mencionaban:

1) la agrupación de los productores consustanciados con las ideas expuestas; 2) la integración de todos los subsectores de la cadena de producción de la carne (productores ganaderos, industrias frigoríficas, agentes de comercialización, etc.); 3) la comercialización grupal y su inmediata consecuencia: la economía en escala; 4) el control o captación de la demanda, mediante la concentración de la oferta (AHMRC, H, $S A$, viernes 18/10/1996, p. 7).

\subsection{Las cámaras asesoras y la búsqueda de las capacidades empresarias}

La prestación de servicios y la capacitación en aspectos técnicos vinculados al agronegocio que desarrollamos en el apartado anterior también implicó, por parte de la SRRC, la promoción entre sus asociados del establecimiento de vínculos y realización de negocios con empresarios nacionales e internacionales. Este servicio adicional brindado por la dirigencia de la SRRC puede ser entendido como parte de la redefinición de sus perfiles institucionales. Estas acciones buscaban fortalecer el perfil empresario de las bases sociales de la entidad para favorecer la participación -sin intermediación- en mercados internacionales. Mario Lattuada (2006) ha visto en este aspecto un indicador que habría posibilitado a las asociaciones de tipo reivindicativo superar las consecuencias del síndrome de competencia. La estrategia de asumir una actitud más propositiva o de infraestructura), viabilizó la redefinición de la relación con las bases sociales, lo que incrementó sus capacidades técnicas y posibilitó un mayor desarrollo de las actividades de promoción, comercialización y servicios.

Como mencionamos, desde la segunda mitad de la década de 1990 la dirigencia de la SRRC propició la estructuración de vínculos con empresarios extranjeros para así robustecer lazos comerciales. Muchas de estas 
experiencias fueron realizadas por intermedio de la Municipalidad de Río Cuarto ${ }^{6}$; otras, en cambio, se desarrollaron a partir de vínculos de los propios productores. A modo de ejemplo, en 1994 se emprendió un viaje de negocios a la ciudad de Abilene, Texas, donde se visitaron establecimientos y se hizo contacto con productores ganaderos de esa región (AHMRC, H, $S A$, viernes 07/10/1994, p. 11). También se realizaron misiones de negocios a Israel a través de las gestiones del cónsul de ese país y el gobierno de la provincia de Córdoba. En esta oportunidad se facilitaba el viaje con el objetivo de asistir a una muestra de tecnología y así poder adquirir maquinarias agrícolas de avanzada, especialmente sistemas de riego y tratamiento de aguas. Asimismo, se discutió la posibilidad de establecer una cooperación por medio de la firma de un joint ventures. Esta estrategia empresarial permitía a una de las partes, la SRRC, acceder a los paquetes tecnológicos ofrecidos por los empresarios israelíes y, a éstos, como contrapartida, tener acceso al mercado argentino (AHMRC, $\mathrm{H}$, $P$, viernes 01/03/1996, p. 22. Ver también: SRRC, Memorias, año 1996). En 1997 un grupo de empresarios japoneses, por medio de las gestiones de una consultora internacional, visitó las empresas agropecuarias de productores asociados a la SRRC con el objetivo de apreciar las formas de producción de novillos a campo abierto. La intención era no solo conocer sobre los costos de campos, de ganado, etc., para hacer evaluaciones posteriores, sino también obtener información que les permitiera sacar conclusiones para realizar eventuales negocios con los productores de la región (AHMRC, H, $S A$, viernes 24/10/1997. p. 7).

Otra de las modalidades por medio de las cuales se procuró fortalecer las actividades comerciales de los asociados a la entidad fue la celebración de rondas de negocios. Estas jornadas realizadas en el lapso de entre uno a tres días funcionaban como un mecanismo para promover contactos a los productores de forma directa con diferentes empresas. En una intervención en la prensa de los dirigentes de la SRRC se daba cuenta de los motivos que impulsaban este tipo de emprendimientos:

...esta Sociedad Rural se viene “aggiornando", procurando abrir caminos al sector que representa, para acometer estos nuevos
desafíos que la actualidad nos impone. Hemos venido bregando, durante muchos años, para que nuestro gobierno propicie
estos acercamientos, pero hemos aprendido que no debemos esperarlo todo de nuestras autoridades y que ha llegado la hora
de darle adecuados cauces a los emprendimientos privados, para desarrollar nuestras potencialidades productivas. En una
palabra, favorecer transacciones, de nivel internacional, que viabilicen el comercio y que ponga nuestra producción en una
mejor posición, en los distintos mercados (...) es un principio y todo dependerá, en definitiva, de la habilidad de nuestra
gente para desenvolverse empresarialmente y crear, por lo menos, bases sólidas para futuras transacciones (AHMRC, H, $S A$,
$01 / 09 / 1995$, p. 7$)$.

Aparecía enunciada en esa columna parte de la estrategia institucional diseñada por la SRRC, una suerte de redefinición de sus funciones que - sin abandonar las instancias de confrontación con el Estado- comenzaba a priorizar una transformación en el perfil de sus bases sociales a partir del fomento al "desenvolvimiento empresario”. En esa apelación, además, quedaban plasmadas las consecuencias del abandono por parte del Estado, de sus funciones de intervención y de redistribución del excedente intersectorial (Lattuada, 2006, p. 177), lo que, en el caso estudiado, implicó la búsqueda de acciones para evitar la erosión de sus bases sociales. La columna continuaba indicando las transformaciones inducidas por el cambio en el sistema agroalimentario, especialmente en la idea de que atender las demandas de un consumidor global implicaba cumplir con condiciones de calidad, y, por ende, la necesidad de proveerse de saberes adecuados para llevar adelante tanto los procesos de elaboración de la materia prima como de su posterior comercialización.

Por otra parte, si es verdad que tenemos que adecuar nuestra producción primaria a las actuales exigencias de los consumidores del mundo, bueno será que sepamos lo que hacen en los países más avanzados en la materia (...) Vendrá posteriormente la etapa de saber vender esa producción, con los aditamentos que el mercado internacional exige. En definitiva, nuestra Sociedad Rural propicia el establecimiento de canales idóneos para favorecer estos acercamientos empresarios, que se constituyen en basamentos necesarios para el despegue comercial de nuestras empresas productivas (AHMRC, H, SA, 01/09/1995, p. 7).

Sobre esos lineamientos, en noviembre de 1995 se realizó una ronda de negocios con porcicultores y representantes de veinte firmas de Dinamarca, Francia y España, interesadas en invertir en emprendimientos 
productivos y en vender tecnología destinada a la instalación de plantas procesadoras de carne. También en julio de 2001 tuvo lugar otra ronda de negocios en la que los productores agropecuarios de la región se acercaron a más de doscientas empresas nacionales dedicadas a la fabricación de maquinaria e implementos agrícolas, y también a empresas productoras de bienes y servicios para el agro. En esta oportunidad oficiaron como organizadores el Instituto de Desarrollo Empresario Bonaerense (IDEB), el CECIS de Río Cuarto y la SRRC (Cfr.: AHMRC, H, P. sábado 14/05/1995, p. 20; AHMRC, H, $S A$, viernes 18/08/1995, p. 7; SRRC, Memorias, año 1995; AHMRC, H, $P$, sábado 14/07/2001; AHMRC, H, $S A$, viernes 20/07/2001, p. 3; SRRC, Memorias, año 2001).

Complementariamente a estas actividades, una de las características que señalamos más arriba fue la siempre presente preocupación por acceder a información tanto sobre el funcionamiento y las perspectivas de los mercados internacionales como a las modalidades de comercialización. Especialmente importantes fueron aquellas que favorecían la integración de diversos actores de la cadena productiva, o bien buscaban la asociación entre productores con el objeto de incrementar los volúmenes comercializables y así lograr mejores perspectivas de ganancias económicas.

La primera de las preocupaciones se canalizó, fundamentalmente, por medio de la organización de charlas y conferencias que sintetizamos en la tabla 1. Este imperativo - presente de forma temprana en los ruralistas riocuartenses- por asirse de conocimientos relativos a la dinámica de los mercados internacionales y de herramientas financieras para lograr mejorar sus perspectivas empresariales fue un indicador característico del nuevo perfil dirigencial proyectado por las asociaciones no reivindicativas (Gras, 2009; Hernández, 2009). La centralidad del conocimiento como componente gravitante en la organización de las empresas agropecuarias implicaba generar (o bien reactualizar) solidaridades con los técnicos y profesionales portadores de los nuevos saberes que el agronegocio colocó en un lugar central. Así, en el transcurso del período analizado, compartieron sus conocimientos con los asociados de la SRRC, ingenieros agrónomos y médicos veterinarios de CREA-zona centro, economistas y licenciados en administración de la Fundación de Investigaciones Económicas Latinoamericana (FIEL), así como asesores de la Bolsa de Comercio de Buenos Aires y de Asociación de Acopiadores de Cereales.

Entre las temáticas abordadas, más allá de las que planteaban los análisis de coyuntura económica, eran significativas las que comunicaban sobre instrumentos financieros para intervenir en mercados internacionales. El warrant y las operatorias en mercados futuros eran los recursos financieros más difundidos dentro de las actividades organizadas por la Rural. Estas alternativas para la comercialización de commodities tenían por finalidad aportar certidumbre a las transacciones de los distintos agentes. Su empleo se extendió principalmente en las ventas de cereales y no pudo prosperar en el mercado de carne vacuna por diferentes motivos, como errores estatutarios, déficit de difusión y cuestiones de oportunidad e incomprensión del sistema por parte de los principales actores (Carreras, 2010, p. 35). 
TABLA 1

Detalle de charlas y conferencias relativas a mercados y herramientas de comercialización organizadas por la SRRC

\begin{tabular}{|c|c|c|c|}
\hline Fecha & Temática & Disertantes & $\begin{array}{c}\text { Entidades } \\
\text { auspiciantes y/o co- } \\
\text { organizadoras }\end{array}$ \\
\hline $\begin{array}{l}\text { Jueves } \\
18 / 10 / 1989\end{array}$ & $\begin{array}{l}\text { Formación de precios y } \\
\text { mercados de granos. Situación } \\
\text { y perspectivas económicas }\end{array}$ & $\begin{array}{l}\text { Alce Davite } \\
\text { (Sociedad de } \\
\text { Acopiadores de } \\
\text { Córdoba) } \\
\text { Raúl Dente } \\
\text { (Asociación de } \\
\text { Acopiadores de } \\
\text { Cereales) } \\
\text { Dr. Raúl Fuentes Rossi }\end{array}$ & $\begin{array}{l}\text { Federación de } \\
\text { Centro y Entidades } \\
\text { Gremiales de } \\
\text { Acopiadores de } \\
\text { Cereales }\end{array}$ \\
\hline $\begin{array}{l}\text { Domingo } \\
08 / 07 / 1990\end{array}$ & $\begin{array}{l}\text { Análisis de costos y } \\
\text { perspectivas de rentabilidad } \\
\text { para la campaña 1990/1991. }\end{array}$ & & \\
\hline $\begin{array}{l}\text { Sábado } \\
21 / 07 / 1990\end{array}$ & $\begin{array}{l}\text { Análisis y perspectivas del } \\
\text { mercado de granos y márgenes } \\
\text { brutos de los cultivos } \\
\text { 1990/1991. } \\
\text { Análisis y perspectivas de } \\
\text { macroeconomía. }\end{array}$ & $\begin{array}{l}\text { Lic. Marcelo Avogadro } \\
\text { Dr. Daniel Artana (FIEL) }\end{array}$ & CREA-zona Centro \\
\hline $\begin{array}{l}\text { Viernes } \\
01 / 11 / 1991\end{array}$ & $\begin{array}{l}\text { Mercado de Futuro y opciones: } \\
\text { una nueva alternativa de } \\
\text { comercialización }\end{array}$ & $\begin{array}{l}\text { Lic. Ignacio Iriarte } \\
\text { (Dir. de Informe } \\
\text { Ganadero y analista } \\
\text { especializado en temas } \\
\text { de ganados y carnes) } \\
\text { Dr. Raúl Fuentes Rossi }\end{array}$ & \\
\hline $\begin{array}{l}\text { Martes } \\
21 / 04 / 1992\end{array}$ & $\begin{array}{l}\text { Comercialización de productos } \\
\text { agropecuarios }\end{array}$ & $\begin{array}{l}\text { Lic. Marcelo Avogadro } \\
\text { Dr. Raúl Fuentes Rossi }\end{array}$ & CREA-zona Centro \\
\hline $\begin{array}{l}\text { Lunes } \\
29 / 06 / 1992\end{array}$ & $\begin{array}{l}\text { La problemática de la } \\
\text { operatoria en los mercados } \\
\text { futuros y opciones. }\end{array}$ & Dr. Santiago Burín & \\
\hline $\begin{array}{l}\text { Miércoles } \\
14 / 10 / 1994\end{array}$ & $\begin{array}{l}\text { Mercados de futuros y } \\
\text { opciones. }\end{array}$ & $\begin{array}{l}\text { Joaquín Ledesma } \\
\text { Carlos Etchepare } \\
\text { (Bolsa de Cereales de } \\
\text { Buenos Aires) }\end{array}$ & $\begin{array}{l}\text { Agrovet } \\
\text { DowElanco }\end{array}$ \\
\hline $\begin{array}{l}\text { Domingo } \\
16 / 07 / 1995\end{array}$ & Comercialización de carnes. & & AACREA \\
\hline $\begin{array}{l}\text { Viernes } \\
31 / 02 / 1997\end{array}$ & $\begin{array}{l}\text { Warrant: para obtener dinero sin } \\
\text { tener que malvender. }\end{array}$ & & \\
\hline $\begin{array}{l}\text { Viernes } \\
18 / 04 / 97\end{array}$ & $\begin{array}{l}\text { Jornada de capacitación y } \\
\text { actualización } \\
\text { Temas: Una visión económica } \\
\text { de mediano plazo sobre el } \\
\text { sector agropecuario. } \\
\text { Diagnóstico y pronóstico de } \\
\text { los fenómenos meteorológico } \\
\text { que inciden en la actividad } \\
\text { productiva. Perspectivas de los } \\
\text { mercados de granos y nuevas } \\
\text { herramientas comerciales. }\end{array}$ & $\begin{array}{l}\text { Dr. Fuentes Rossi } \\
\text { Dr. Federico Norte } \\
\text { Cr. Daniel Miró } \\
\text { Cr. Héctor Niell }\end{array}$ & \\
\hline $\begin{array}{l}\text { Martes } \\
29 / 04 / 1997\end{array}$ & $\begin{array}{l}\text { Perspectivas del mercado } \\
\text { ganadero. }\end{array}$ & Dr. Raúl Fuentes Rossi & \\
\hline $17 / 10 / 1997$ & $\begin{array}{l}\text { La crisis internacional y su } \\
\text { incidencia sobre los sectores } \\
\text { de la producción. Perspectivas } \\
\text { para el sector agropecuario. }\end{array}$ & Dr. Raúl Fuentes Rossi & \\
\hline $\begin{array}{l}\text { Viernes } \\
12 / 05 / 2000\end{array}$ & Operatoria de mercados & & \\
\hline
\end{tabular}

Fuente: elaboración propia sobre la base de notas periodísticas y memorias institucionales para el período 1988-2002 Nota: en la tabla no están reflejadas las charlas y conferencias desarrolladas en el marco de las ferias organizadas por la entidad 
Otra inquietud de la dirigencia ruralista fue generar instancias asociativas para mejorar la capacidad empresaria de sus productores asociados y, paralelamente, reforzar la acción gremial de la entidad. Así, por un lado, a través del médico veterinario Edgar Mondino y del ingeniero agrónomo Pedro Rinaudo, miembros de la SRRC y representantes de la cámara de cerdos de la entidad, se impulsó la constitución de la delegación regional de la Asociación Argentina de Criadores de Cerdos. El objetivo radicaba en enfrentar algunos problemas por los que atravesaba esa actividad productiva, especialmente derivados de la "falta de transparencia del mercado que ocasiona distorsiones en la comercialización" y de las "falencias en los controles sanitarios" ${ }^{7}$. Por otro lado, también se impulsó la asociación de productores para comprar y operar con maquinaria agrícola a través de la reunión de entre cuatro a diez productores con la finalidad de realizar las actividades de labranza y conservación apropiada de los forrajes. Se buscaba de esa forma dar solución a las restricciones de capital y a la obsolescencia de las maquinarias agrícolas empleadas (AHMRC, $\mathrm{H}, S A$, viernes 29/10/1993, p. 7).

Asimismo, desde la conducción de la SRRC se emprendieron acciones -más o menos exitosas- para crear seguros de inversión agrícola y brindar servicios de venta de agrodiesel para los asociados. Se fomentaron, además, diversas iniciativas para la generación de empresas agroindustriales (AMRC, H, SA, viernes 19/03/1993, p. 2; AHMRC, H, $S A$, viernes 18/08/2000, p. 11). Un claro ejemplo de esta última situación fue la erección de una planta industrial de productos lácteos en la localidad de San Basilio, que poseía una capacidad inicial para procesar 25.000 litros de leche diarios, la cual podía alcanzar un volumen de hasta 50.000 litros (SRRC, Memorias, año 1997; AHMRC, H, $P$, viernes 08/11/1997, p. 12). El emprendimiento reunía a treinta productores lácteos de la región, la mayoría socios de la SRRC, que:

Tras sufrir innumerables vaivenes y altibajos en sus empresas, siempre supeditadas a políticas comerciales no suficientemente claras y convenientes, decidieron un día acometer por medio de la acción de esta Sociedad Rural la empresa de asociarse para procesar ellos mismos la materia prima que producen en sus campos y así obtener mejores condiciones para alcanzar los objetivos que los identifica, que los alienta: mejorar sus sistemas de producción y a la vez mejorar también la necesaria rentabilidad de sus empresas. Teniendo claro el diagnóstico de situación y claras perspectivas de desarrollo inmediato, realizaron los necesarios estudios de mercado, sobre la base de inversiones escalonadas (AHMRC, $\mathrm{H}, S A$, viernes 28/11/1997, p. 7).

Estas estrategias, que buscaban fortalecer la empresarialidad de los productores del sur cordobés, parecían estar atadas a la inquietud e iniciativa de las cámaras de la entidad (la de porcinos y de lechería fueron las que se mostraron más activas junto con la de granja y producciones alternativas), y, por lo tanto, estaban más dirigidas a un grupo reducido de asociados a la entidad que a constituir el designo de una política institucional de la SRRC, aunque sí se pueden enmarcar en todo un contexto institucional que fomentaba este tipo de acciones.

\section{A MODo DE CONCLUSión}

El contexto productivo de la década de 1990 estuvo signado por una serie de rupturas respecto de períodos anteriores. Tal vez una de las más visibles fue la emergencia de entidades centradas en la difusión de los saberes y en el manejo de herramientas consideradas indispensables para transitar exitosamente los procesos productivos. La presencia de estas entidades complejizó y fragmentó aún más la ya heterogénea estructura asociativa del agro pampeano. Las dinámicas generadas por estos actores no implicaron un desplazamiento de las asociaciones gremiales que tradicionalmente habían dominado la representación de intereses agrarios en períodos históricos previos. Por el contrario, supuso para muchas de ellas la implementación de un conjunto de estrategias que tensionaron las funciones que históricamente las habían caracterizado. 
En ese sentido, por medio del análisis de la SRRC pudimos observar cómo la dirigencia rural introdujo un conjunto de modificaciones a su modelo organizativo, las cuales no solo se plasmaron estatutariamente sino que generaron una nueva forma de concebir la actividad gremial. Las denominadas cámaras asesoras, pensadas como un espacio institucional de circulación de saberes entre la dirigencia rural, los técnicos y las bases sociales de la entidad constituyeron una respuesta a los imperativos del agronegocio. Profesionalizar la actividad agropecuaria, es decir, brindar diversas capacidades (productivas, organizacionales, informáticas, financieras, etc.) a los productores para adecuar sus prácticas al contexto del agronegocio fue el horizonte institucional trazado por la dirigencia desde mediados de la década de 1990. Asimismo, este nuevo rumbo implicó la posibilidad de concertar negocios, de acceder a otros mercados, de eliminar la intermediación y establecer una relación directa con el mercado internacional. Profesionalizar la actividad agropecuaria para estos actores fue, en definitiva, potenciar la capacidad empresaria de sus bases sociales.

El análisis propuesto invita a continuar interrogándose sobre la dinámica de la estructura asociativa del agro pampeano. Es decir, lejos de pensar como lógicas excluyentes las dinámicas de las entidades técnicas o de "nuevo cuño" y las de las devenidas "tradicionales" o gremiales, es necesario avanzar en la complementación que generaron. De esta forma, podemos observar la emergencia de un estilo gremial distinto que sin desatender las instancias de mediación política frente al Estado (en todos sus niveles) procuró acercar los saberes y habilidades del nuevo contexto productivo a sus asociados. Esta operación, a su vez, invita a repensar no solo las escalas desde las cuales observamos los fenómenos, sino también las categorías que implementamos para dar cuenta de los mismos.

\section{REFERENCIAS BIBLIOGRÁFICAS}

Alapin, H. (2008). Rastrojos y algo más. Historia de la siembra directa en Argentina. Buenos Aires: Teseo/Universidad de Belgrano.

Barsky, O. y Gelman, J. (2009 [2001]). Historia del agro argentino. Desde la conquista hasta comienzos del siglo XXI. Sudamericana: Buenos Aires.

Bocchicchio, A. y Cattáneo, C. (2005). Transformaciones en la agricultura e innovación organizacional en asociaciones de productores: los casos de AAPRESID y ASAGIR. En R. Benencia y C. Flood, (Comps.), Trayectorias y contextos. Organizaciones rurales en la Argentina de los noventa (pp. 89-104). Buenos Aires: La Colmena.

Carini, G. (2015). Nuevo Estado, viejos intereses: corporaciones agrarias y mediación política en el interior de Córdoba (1935-1955). Estudios del ISHIR. 5(11), 93-113. Recuperado de: http://web2.rosario-conicet.gov.ar/ojs/index .php/revistaISHIR/index

Carini, G. (2017). Estado, asociaciones de productores y agronegocios: dinámicas locales y redefinición de perfiles institucionales. Coordenadas. Revista de Historia Local y Regional, 4(1), 219-239. Recuperado de: http://ppct.c aicyt.gov.ar/index.php/coordenadas/article/view/11220/9979

Carini, G. (2017a). "Cada día más empresarios": asociaciones de productores, agronegocio y estrategias institucionales en la pampa cordobesa (1995-2002)”. Sociohistórica. Cuadernos del CISH, (40), 1-20. Recuperado de: http://w ww.sociohistorica.fahce.unlp.edu.ar/article/view/SHe032/8969

Carini, G. (2018). Agro, negocio y nueva institucionalidad en las pampas: itinerarios y propuestas de abordajes para el análisis de la representación de intereses agrarios. En G. Banzato, G. Blanco y J. Perrén (Comps.), Expansión de la frontera productiva y estructura agraria argentina, siglos XIX-XXI (pp. 417-439). Buenos Aires: PrometeoAsociación Argentina de Historia Económica.

Carini, G. (2018a). Agronegocio, entidades agrarias y redefinición de perfiles institucionales en la pampa cordobesa (fines del siglo XX). Pilquen. Sección Ciencias Sociales, 2(21), 83-94. Recuperado de: http://revele.uncoma.edu. ar/htdoc/revele/index.php/Sociales/article/view/1907

Carreras, A. (2010). Ganados y carnes vacunas. En L. Reca, D. Lema y C. Flood (Eds.), El crecimiento de la agricultura argentina. Medio siglo de logros y desafíos (pp. 29-66). Buenos Aires: Editorial de la Facultad de Agronomía de la Universidad de Buenos Aires. 
Flood, C. (2005). Trayectorias institucionales comparadas de innovación tecnológica en la región pampeana. En R. Benencia y C. Flood (Comps.), Trayectorias y contextos. Organizaciones rurales en la Argentina de los noventa (pp. 137-177). Buenos Aires: La Colmena.

Gallacher, M. (2010). Cambios en la asignación de recursos entre agricultura y ganadería. En L. Reca, D. Lema y C. Flood (Eds.), El crecimiento de la agricultura argentina. Medio siglo de logros y desafios (pp. 1-29). Buenos Aires: Editorial de la Facultad de Agronomía de la Universidad de Buenos Aires.

Gras, C. (2009). El nuevo empresariado agrario: sobre la construcción y los dilemas de sus organizaciones. En C. Gras y V. Hernández (Coords.), La Argentina rural. De la agricultura familiar a los agronegocios (pp. 215-237). Buenos Aires: Biblos.

Gras, C. y Hernández, V. (2009). El fenómeno sojero en perspectiva: dimensiones productivas, sociales y simbólicas de la globalización agrorrural en la Argentina. En C. Gras y V. Hernández (Coords.), La Argentina rural. De la agricultura familiar a los agronegocios (15-39). Buenos Aires: Biblos;

Gras, C. y Hernández, V. (2013). Los pilares del modelo agribusiness y sus estilos empresariales. En C. Gras y V. Hernández (Coords.), El agro como negocio. Producción, sociedad y territorios en la globalización (pp. 17-48). Buenos Aires: Biblos.

Gras, C. y Hernández, V. (2013a). Asociatividad del empresariado agrícola en Argentina. AACREA y AAPRESID en perspectiva. En J. Muzlera y A. Salomón (Coords.), Sujetos sociales del agro argentino. Configuraciones históricas y procesos de cambio (pp. 36-67). Rosario: Prohistoria.

Gras, C. y Hernández, V. (2016). Radiografía del nuevo campo argentino Del terrateniente al empresario transnacional. Buenos Aires: Siglo XXI.

Hernández, V. (2009). La ruralidad globalizada y el paradigma de los agronegocios. En C. Gras y V. Hernández (Coords.), La Argentina rural. De la agricultura familiar a los agronegocios (pp. 39-65). Buenos Aires: Biblos.

Lattuada, M. (2006). Acción colectiva y corporaciones agrarias en la Argentina. Transformaciones institucionales a fines del siglo XX. Bernal: Universidad Nacional de Quilmes.

Liaudat, M. (2015). La construcción hegemónica de las entidades técnicas en el agro argentino: análisis de los discursos de AAPRESID y AACREA en la última década. Mundo Agrario, 16(32), 1-32. Recuperado de: http://www.m undoagrario.unlp.edu.ar/article/view/MAv16n32a04

Murmis, M. (1998). Agro argentino: algunos problemas para su análisis. En N. Giarracca y S. Cloquell (Comps.), Las agriculturas del Mercosur. El papel de los actores sociales (pp. 205-211). Buenos Aires: La Colmena.

Palomino, M. (1988). Tradición y poder: La Sociedad Rural Argentina (1955-1983). Buenos Aires: CISEA-Grupo Editor Latinoamericano.

Teubal, M., Domínguez, D. y P. Sabatino (2005). Transformaciones agrarias en la Argentina. Agricultura industrial y sistema agroalimentario. En N. Giarraca y M. Teubal (Coords.), El campo argentino en la encrucijada. Estrategias y resistencias sociales, ecos en la ciudad (pp.37-78). Buenos Aires: Alianza.

\section{Notas}

1 Muchos especialistas han observado cómo a partir de mediados de la década de 1990 se configuró un nuevo modelo agropecuario. Así, desde diferentes perspectivas la forma que asumió la organización del trabajo agropecuario fue denominada, por ejemplo, como "una agricultura sin agricultores" (Teubal et al., 2005), con énfasis en las consecuencias que el modelo ocasionó sobre la composición de la estructura agraria. En cambio otros, sin desconocer estos alcances, procuraron definiciones más comprensivas para referirse a esta etapa del desarrollo agropecuario. En ese sentido, aquí usamos la idea de agronegocio o ruralidad globalizada empleada por Gras y Hernández (2009). Estas definiciones ponen el acento en la reorganización de los sistemas productivos, que transforman el capital fijo en variable e intensifican la tercerización de labores y la contratación de la tierra. Asimismo, en ese entramado se incorpora como factor determinante el conocimiento. Es decir, la mayor eficacia ya no deriva solo de las prácticas agronómicas sino también de las vinculadas al gerenciamiento. Esto conlleva una ruptura en la forma en que era entendido el "oficio" de productor y la actividad agropecuaria en general hasta ese momento. (Gras y Hernández, 2009, 2013 y 2016). Para una arqueología conceptual puede consultarse Hernández (2009). 
2 Existe una considerable cantidad de investigaciones que se han ocupado de analizar los modelos organizativos de las entidades de nuevo cuño. Entre las más destacadas podemos mencionar la de Flood (2005), que ofrece un panorama referido a la nueva institucionalidad con hincapié en la Asociación Argentina de Productores de Siembra Directa (AAPRESID). Bocchicchio y Cattáneo (2005) propusieron una mirada comparativa por diversas dimensiones institucionales entre la AAPRESID y la Asociación Argentina del Girasol (ASAGIR). También sobre AAPRESID puede consultarse el trabajo de Alapín (2009). En diversos trabajos de Gras (2009), Gras y Hernández (2013a) han ofrecido un exhaustivo recorrido por las lógicas institucionales de la Asociación Argentina de Consorcios Regionales de Experimentación Agrícola (AACREA), y, en menor medida, de la AAPRESID. También Liaudat (2015) se ocupó de reconstruir las discursividades de estas entidades.

3 Algunas implicancias analíticas referidas a las miradas sobre las asociaciones agropecuarias ("técnicas" y "tradicionales") durante el agronegocio las realizamos en Carini (2018), a donde remitimos.

4 Cabe advertir que la acción gremial de la SRRC se asentaba en la ciudad de Río Cuarto, cabecera del departamento homónimo. Esta agrociudad, ubicada al sur de la provincia de Córdoba, brinda una serie de bienes y servicios que se presentan como significativos para el funcionamiento del sector agropecuario y proyectan su influencia más allá de los límites departamentales. El espacio económico del departamento de Río Cuarto se caracterizó por la presencia de un sistema productivo mixto con predominio de la ganadería, dado que más de un $70 \%$ del suelo se destinaba a esa actividad. Hacia mediados de la década de 1990 esta configuración comenzó a sufrir transformaciones por causa del auge de la soja y de la implementación de las diversas innovaciones productivas que dicho auge implicó. Como en otras regiones, hubo un crecimiento exponencial del cultivo de soja -en 2002 registró un crecimiento del 82 \% en la superficie implantada- que supuso una disminución correlativa de las forrajeras, las cuales pasaron a ocupar la mitad de la superficie implantada que poseían en 1988. A pesar de esta situación, el stock ganadero se mantuvo relativamente estable. Esto implica importantes cambios en la actividad pecuaria que se manifestaron en las innovaciones respecto de las formas de organizar el trabajo dentro de la explotación agropecuaria y de las innovaciones productivas que se implementaron. Asimismo, hubo un pronunciado proceso de liquidación de explotaciones agropecuarias: se pasó de 4580 en el año 1988 a 2984 explotaciones agropecuarias en 2002, lo que implicó una disminución de casi el $35 \%$, superior a la media provincial y nacional. La escala promedio de las explotaciones pasó de 371,91 hectáreas en 1988 a 492,05 hectáreas en 2002, lo que implica un crecimiento de un $32,30 \%$. Este es uno de los elementos que permiten observar -junto a la adopción del paquete tecnológico- el pasaje a un esquema de capital intensivo, el cual demandó nuevas escalas de operaciones (Carini, 2017).

5 Esta publicación circulaba los días viernes y presentaba características bastante uniformes para el período que va entre mayo de 1995 y enero de 2002: su estructura o conjunto de secciones no varió, y la cantidad de páginas osciló entre 8 y 12 de tamaño tabloide. Los promedios anuales por edición de la circulación neta pagada de los días viernes para el período considerado fueron de 6.197 ejemplares (datos proporcionados por el Instituto Verificador de Circulaciones - IVC). Los entramados discursivos asociados a la difusión y legitimación de los imperativos del agronegocio mediatizados por medio de esta columna los reconstruimos en Carini (2017a).

6 Aunque en las fuentes no se hace explícito, creemos que esas actividades se realizaban en el marco de un convenio firmado entre la Municipalidad de Río Cuarto y la SRRC con la finalidad de desarrollar emprendimientos conjuntos de base agrícola y ganadera para fomentar las actividades productivas de la región. Esto implicaba tanto el aspecto productivo como el comercial, correspondiente a los canales de comercialización y a la búsqueda de mercados. SRRC, Memorias, año 1994 y SRRC, Memorias, año 1995.

7 Cfr.: AHMRC, H, $P$, jueves 29/10/1992, p. 26; AHMRC, H, $P$, domingo 08/11/1992, p. 24; AHMRC, H, $P$, martes 17/11/1992, p. 28; AMRC, H, $S A$, viernes 20/11/1992, p. 3. No pudimos indagar demasiado en el desenvolvimiento posterior de esta iniciativa; sí encontramos referencias a la actuación en la cámara de la entidad, especialmente en la oportunidad de la organización de la feria anual de otoño. 